\title{
Estilos de aprendizagem em ambientes virtuais: cenários de investigação na educação superior
}

\author{
MARCOS ANDREI OTA ${ }^{\mathrm{i}}$ \\ Universidade Cruzeiro do Sul, Brasil \\ ota@cruzeirodosulvirtual.com.br
}

\author{
CARLOS FERNANDO ARAUJO JÚNIOR \\ Universidade Cruzeiro do Sul, Brasil \\ carlos.araujo@cruzeirodosul.edu.br
}

\author{
DANIELA BARROS \\ Universidade Aberta, Portugal \\ dmelare@gmail.com
}

RESUMO: Este estudo propõe uma breve investigação de trabalhos acadêmicos e científicos em língua portuguesa no período de 2010 a 2016 que abordaram a convergência dos fundamentos das teorias de estilo de aprendizagem em ambientes virtuais no contexto do ensino superior. Trata-se de um estudo exploratório, com análise descritiva suportada por referenciais bibliográficos. A coleta de dados foi realizada nos principais repositórios portugueses e brasileiros. Um total de 46 produções foram encontradas diante dos critérios de busca estabelecidos. Para compor a análise dos resultados, utilizou-se o critério da dimensão organizacional e categorização dos trabalhos por foco e sub-foco temáticos. Dos resultados obtidos, destaca-se o mapeamento sobre as investigações que relacionam as teorias dos estilos de aprendizagem com a educação a distância. Relativamente aos resultados, percebe-se que há um esforço das investigações que buscam reunir contribuições e práticas pedagógicas que possam se aproximar das necessidades específicas dos estudantes matriculados em cursos superiores a distância.

Palavras-chave: Estilos de Aprendizagem; Ambientes Virtuais; Educação a Distância; Educação Superior; Mapeamento de Pesquisa

\section{INTRODUÇÃO}

A educação a distância $(\mathrm{EaD})$ é uma modalidade de ensino em constante transformação e expansão pois representa parte de um processo de inovação educacional mais amplo que é a integração das novas tecnologias digitais nos processos educacionais (Belloni, 2002). As tentativas com a modalidade a distância acumulam ações desde o século passado, com experiências a partir de cursos profissionalizantes por carta, rádio e TV. O que diferencia a $\mathrm{EaD}$ praticada hoje daquela praticada tempos atrás são aos meios disponíveis e adequados em cada época (Dias, 2010).

Se forem considerados os últimos dez anos, o número de matriculados nos cursos superiores a distância no Brasil passou de 5 mil para 30 mil (Censo EaDBR, 2015). Estes dados indicam que 1 em cada 6 ingressantes matriculados, consideram a educação a distância uma alternativa mais acessível e garantida de estudo. O aumento acelerado desses cursos impõe às instituições de ensino superior (IES), novas funções 
e desafios, exigentes de redimensionamento de currículos, práticas pedagógicas e tecnologias.

Adaptar-se às peculiaridades dos alunos faz da EaD uma opção incontestável para assegurar a continuidade dos estudos e proporcionar um ensino de qualidade mesmo em áreas remotas, tudo isso graças aos avanços na inclusão digital, seja pela criação da banda larga popular ou pela diminuição dos custos de acesso à Internet.

Essas tendências têm levado instituições de ensino superior a repensarem sobre sua representatividade e importância nesse cenário e consequentemente, buscarem soluções para ampliar as possibilidades de oferta de cursos, melhorias didático-pedagógicas e modelos educacionais que caminhem na mesma direção das transformações e exigências de mercado. A exemplo disso, nota-se um número relevante de estudos adotando as contribuições das teorias dos estilos de aprendizagem como forma de disseminar concepções educacionais inovadoras que se conectem cada vez mais às preferências dos estudantes, aos aspectos que integram as relações do saber (ensino e aprendizagem) nos ambientes virtuais de aprendizagem (AVAs) seja de apoio ao modelo presencial, híbrido e/ou totalmente a distância (Santos; Bariani \& Cerqueira, 1999 ; Lopes, 2002; Barros, 2009-2011; Freitas, 2013).

A motivação desse trabalho ancora-se pela oportunidade de conceder acesso a um mapeamento inicial por focos temáticos (Fiorentini,1994, 2002) de publicações que recorreram às teorias dos estilos de aprendizagem na tentativa de compreender as preferências de estudantes em modelos de cursos suportados por espaços virtuais .

Com base nos pressupostos apresentados, o presente artigo tem por objetivo apresentar uma breve investigação de trabalhos acadêmicos e científicos em língua portuguesa no período de 2010 a 2016 que abordaram a convergência os fundamentos das teorias de estilo de aprendizagem em ambientes virtuais no contexto do ensino superior.

\section{DESENVOLVIMENTO}

A contextualização teórica restringe-se à triangulação da pesquisa (Figura I) proposta no objetivo deste estudo a fim de gerar um mapeamento dos cenários de publicações que relacionaram os estilos de aprendizagem em ambientes virtuais de cursos superiores.

FigURA I - Triangulação da pesquisa

Fonte: elaborado pelos autores

\section{Estilos de Aprendizagem}

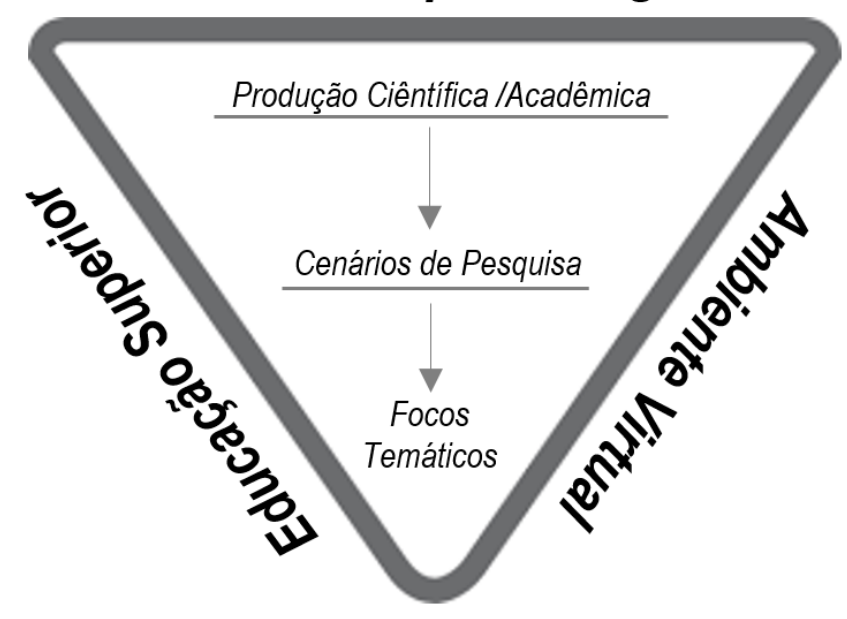

O crescimento do ensino a distância e surgimento de novos recursos midiáticos para promover situações de ensino e aprendizagem em AVA, provocam alterações significativas no cenário do ensino superior. Diante da triangulação apresentada, percebe-se nas leituras/fichamentos realizados que professores, alunos e as IES são naturalmente direcionados a desempenhar novas funções. 
As possibilidades das práticas pedagógicas nesse cenário se ampliam quando é possível identificar nos AVAs das instituições de educação superior, elementos que vislumbrem modelos de aprendizagem colaborativos, proporcionados por uma mediação pedagógica geradora de autonomia e motivações para aprendizagem individual e coletiva (Ota, 2011, p.40)

Varela (2011) salienta que a universidade deve incentivar o processo de criação e renovação do conhecimento. Masetto (2003) adiciona a esse pensamento, uma necessidade das instituições de ensino superior impulsionar a convergência de outras fontes de produção de conhecimento e de pesquisa. Segundo Galvis (1992, p. 52), "um ambiente de aprendizagem poderá ser muito rico, porém, se o aluno não desenvolve atividades para o aproveitamento de seu potencial, nada acontecerá".

Leite filho et al (2008) consideram importante reconhecer as características peculiares de perfil e de estilos de aprendizagem para conseguir propor novas metodologias de ensino, aprendizagem e avaliação, apropriadas às especificidades apresentadas.

Esse "aluno virtual" protagoniza uma série de mudanças de comportamento e ações didático-metodológicas no cenário educacional superior, pois já nasceu num tempo, onde as novas tecnologias já se faziam acontecer. Dessa forma, o centro do "processo de ensino e aprendizagem não é mais o interesse do professor da disciplina, mas o que o aluno precisa aprender" (Maia \& Mattar, 2007, p. 83).

\subsection{Estilos de Aprendizagem em AVA de cursos superiores}

Numa definição simplória, pode-se compreender que os estilos de aprendizagem são formas pelas quais as pessoas aprendem. Há um vasto acervo de publicações e instrumentos para diagnosticar os estilos de aprendizagem. Destacam-se Kolb (1984), Felder \& Silveman (1988), Neil Fleming (VARK-LEARN, 2006) e Alonso, Galego y Honey (2002).
Barros (2009) esclarece que os estilos de aprendizagem não configuram métodos/metodologias com instruções para desenvolver novos saberes, relacionam-se com as preferências inerentes a cada aprendiz, logo, interferem no modo como uma pessoa processa um novo conhecimento.

Dunn (1989) definiu três componentes para tais estilos: 1) a maneira com que se processa a informação; 2) seleção dinâmica de estratégias de aprendizagem; 3) a própria percepção da pessoa com respeito a sua aprendizagem.

Reunir contribuições dos estilos de aprendizagem para entender como promover melhores práticas pedagógicas e situações de aprendizagem nos ambientes virtuais, apresenta-se como um interesse comum entre pesquisadores de muitos países, não apenas pelo número expressivo de trabalhos já publicados, mas também por necessidades peculiares das IES identificarem indicadores de qualidade para oferta de cursos, tratando-se de instituições privadas, a preocupação tende a aumentar com relação à competitividade de mercado e ações para retenção dos alunos.

A exemplo disso, o Ministério da Educação (MEC), a entidade reguladora de cursos superiores a distância, em atenção às potencialidades e ao mesmo tempo às fragilidades dos modelos de EaD, elaborou um documento intitulado: Referenciais de Qualidade para a Educação Superior a Distância, cujo objetivo é indicar os parâmetros que os estudantes necessitam saber antes de se matricularem em cursos nãopresenciais. O documento com os referenciais destaca os seguintes elementos:

- Métodos de ensino;

- Tecnologias;

- Tipo de material didático; 
- Interações disponíveis;

- Tempo de feedback do professor-tutor responder às dúvidas.

Os AVA permitem viabilização de atividades reflexivas e colaborativas, porém a existência de seus recursos, por si só, não garantem o desenvolvimento de ações dessa natureza. Prado e Almeida (2003) entendem que as tecnologias digitais presentes nos espaços virtuais não configuram a eficácia da qualidade do curso e/ou do aprendizado. Para os autores, são os profissionais envolvidos com o planejamento e a execução pedagógica do curso que potencializam o uso desses recursos por meio de criação e recriação de estratégias apropriadas.

O conhecimento do estilo de aprendizagem do indivíduo possibilita o desenvolvimento de potencialidades com maior eficiência e diagnóstico das dificuldades de aprendizado (Sanchez, 2011). DeAquino (2007) alerta para o fato de que o desconhecimento dos diferentes estilos de aprendizagem pode fragilizar a relação entre ensinar e aprender. Tratandose do ensino superior, não considerar esse fato pode comprometer os resultados para a formação do estudante adulto, além de gerar situações de insatisfação e evasão.

\section{Metodologia}

Sob o aspecto metodológico adotado, este artigo caracteriza-se como uma pesquisa de cunho exploratório, com análise descritiva suportada por referenciais bibliográficos. Romanowski \& Ens (2006) citam que esses trabalhos, não se restringem a identificar a produção, mas analisá-la, categorizá-la e revelar os múltiplos enfoques e perspectivas.

Para iniciar o mapeamento dos dados, submeteu-se a organização metodológica em duas etapas: a escolha dos princinpais repositórios em língua portuguesa e estabelecimento de alguns critérios de busca.

\section{Fonte de Coleta de Dados}

- RCAAP - Repositório Científico de Acesso Aberto de Portugal

- OASISbr - Portal Brasileiro de Acesso Aberto à Informação Científica

- CAPES - Periódicos

- RepositóriUM - Repositório institucional da Universidade do Minho

- Revista de Estilos de Aprendizagem

- Repositório institucional da Universidade Aberta (UAB)

\section{Critérios de Busca}

- Consulta dos termos em língua portuguesa, tendo em vista o crescimento exponencial de oferta de cursos a distância em países lusófonos.

- Delimitação dos descritores (título, assunto, resumo) diante dos termos: estilo de aprendizagem ; ambientes virtuais e/ou ensino superior e/ou educação a distância.

- Tipo de produção científica e acadêmica: capítulo de livro, artigos para congressos/conferências, revistas científicas, dissertação de mestrado e teses.

Os critérios adotados tanto na fonte de dados quanto nos critérios de busca, estão intrinsicamente relacionados ao objetivo desse estudo: apresentar uma breve investigação de trabalhos indexados nos referidos repositórios que utilizaram a triangulação (Estilo de Aprendizagem > Ambientes Virtuais > Ensino Superior). A priori, obtivemos um total de 216 trabalhos, entretanto, após adotar os critérios de busca (Figura II) restringimos o nosso estudo a um total de 46 publicações (2010 a 2016), conforme identificação no apêndice deste artigo. 
Figura II - Descritores de Busca

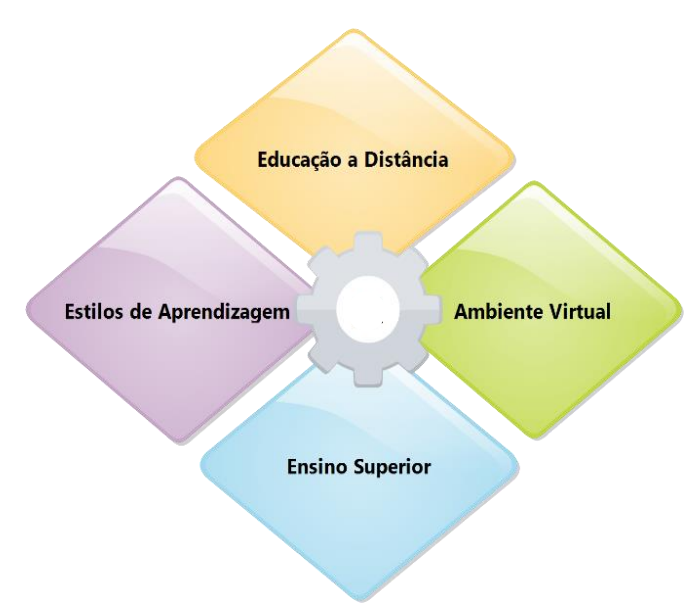

A saber, optou-se por não incluir trabalhos em 2017, pois o levantamento dos dados ocorreram de janeiro a março desse mesmo ano.

Para organização e distribuição das produções no mapeamento proposto (Tabela 1), utilizaram-se os softwares Mendeley e Excel para o registro das observações, fichamento das leituras e categorização dos focos temáticos identificados. Tal estratégia permitiu otimizar os processos de análise dos dados, além de reduzir e delimitar os trabalhos encontrados, conforme a triangulação proposta.

TABELA I - Distribuição das produções

\begin{tabular}{|l|c|}
\hline \multicolumn{2}{|c|}{ Produções Científicas } \\
\hline Revistas Científicas & 11 \\
\hline Congressos/Conferências & 12 \\
\hline Capítulos de Livro & 3 \\
\hline \multicolumn{2}{|c|}{ Produções Acadêmicas } \\
\hline Dissertação de Mestrado & 10 \\
\hline Tese & 10 \\
\hline Total & 46 \\
\hline
\end{tabular}

Nesta direção, consideram-se os seguintes dados:

a) Dados descritivos: título do artigo, palavras-chave, resumo, ano de publicação, área de concentração, origem.

b) Dados temáticos: foco temático, objetivos, metodologia, teorias, contribuições e resultados.

Nos casos em que os dados não foram suficientes para interpretação do trabalho, evidenciados nos resumos, procedeu-se uma leitura ampliada do artigo, com intuito identificar os dados temáticos.

Para Ferreira (2002) esse tipo de pesquisa possibilita evidenciar tendências, apontar caminhos que vêm sendo tomados, além de reunir contribuições para organização e análise na definição de uma determinada área.

Romanowski e Ens (2006) salientam que os dados emergidos de um mapeamento possibilitam uma visão geral das produções na área, além de permitir aos pesquisadores identificarem transformações e lacunas existentes.

Diante dos dados, foi possível identificar 04 focos temáticos e 10 subfocos que serão discutidos na seção seguinte.

\section{RESUltados E DiscuSSões}

Com base nas contribuições propostas por Fiorentini (1994, 2002), os resultados atingidos com a categorização por focos temáticos e seus respectivos subfocos, possibilitam uma visão geral do cenário pretendido. A análise dos dados, num primeiro momento, dedicou-se a tabular os trabalhos a partir da triangulação definida nos aspectos metodológicos da pesquisa. As publicações foram distribuídas por ano e origem (Tabela II). Para o autor, o processo de categorização não é algo simples: 
[...] acontece de forma indutiva e, às vezes, dedutiva, exigindo ajustes individuais (para cada estudo) e grupais (envolvendo um conjunto de estudos). A vantagem é que as categorias construídas emergem do material sob análise e não da literatura propriamente dita, embora, neste processo, o diálogo com a literatura e outras formas de classificação seja conveniente e necessário (Fiorentini, 2002, p.4-5).

TABELA II - Trabalhos selecionados por ano

\begin{tabular}{|c|c|c|c|c|c|c|c|c|c|}
\hline \multirow[t]{2}{*}{ Base de Dados } & \multirow{2}{*}{$\begin{array}{c}\mathbf{N}^{0} \\
\text { artigos }\end{array}$} & \multicolumn{7}{|c|}{ Trabalhos selecionados } & \multirow[t]{2}{*}{ Total } \\
\hline & & 2010 & 2011 & 2012 & 2013 & 2014 & 2015 & 2016 & \\
\hline RCAAP & 11 & 0 & 0 & 4 & 2 & 1 & 0 & 4 & \multirow{6}{*}{46} \\
\hline OASISbr & 16 & 0 & 0 & 4 & 6 & 6 & 0 & 0 & \\
\hline RepositóriUM & 3 & 0 & 0 & 0 & 1 & 1 & 1 & 0 & \\
\hline $\begin{array}{l}\text { Revista Estilos } \\
\text { Aprendizagem }\end{array}$ & 6 & 2 & 1 & 1 & 1 & 0 & 1 & 0 & \\
\hline $\begin{array}{l}\text { Periódicos } \\
\text { CAPES }\end{array}$ & 1 & 0 & 0 & 1 & 0 & 0 & 0 & 0 & \\
\hline $\begin{array}{l}\text { Repositório } \\
\text { UAB }\end{array}$ & 9 & 0 & 1 & 1 & 6 & 0 & 0 & 1 & \\
\hline
\end{tabular}

Conforme estipulado, a segunda e última etapa de análise dos dados, buscou categorizar as produções analisadas nos focos temáticos mapeados. A Tabela 3 apresenta a distribuição dos trabalhos por temática. A coluna "ID" é o mapeamento dos artigos (apêndice) distribuídos conforme o foco e subfoco temático.

TABELA III - Categorização das produções por foco temático

\begin{tabular}{|c|c|c|c|c|}
\hline \multicolumn{5}{|c|}{ Cenários de Investigação (Estilo de Aprendizagem X Ambientes Virtuais) } \\
\hline Foco Temático & 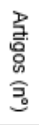 & Subfocos & 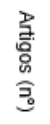 & ID \\
\hline \multirow{3}{*}{$\begin{array}{l}\text { Teorias de Estilo } \\
\text { e metodologias }\end{array}$} & \multirow{3}{*}{19} & Validação de Questionários de Estilo & 10 & $\begin{array}{l}\text { T8, } \mathrm{T} 10, \mathrm{~T} 15, \mathrm{~T} 16, \mathrm{~T} 23, \mathrm{~T} 26, \\
\text { T27, T29, T34, T42* }\end{array}$ \\
\hline & & Parâmetros para escolha e avaliação do curso & 4 & $\mathrm{~T} 14, \mathrm{~T} 36, \mathrm{~T} 39, \mathrm{~T} 42^{*}$ \\
\hline & & Relação do estilo docente com o estilo do aluno & 5 & $\mathrm{T6}, \mathrm{T} 19, \mathrm{~T} 24, \mathrm{~T} 33, \mathrm{~T} 46$ \\
\hline \multirow{2}{*}{$\begin{array}{l}\text { Estratégia de } \\
\text { Ensino }\end{array}$} & \multirow[b]{2}{*}{9} & Planejamento dos objetivos de Aprendizagem & 2 & $\mathrm{~T} 32, \mathrm{~T} 40$ \\
\hline & & $\begin{array}{r}\text { Práticas pedagógicas no contexto da educação a } \\
\text { distância }\end{array}$ & 7 & $\mathrm{~T} 1, \mathrm{~T} 2, \mathrm{~T} 3, \mathrm{~T} 17, \mathrm{~T} 21, \mathrm{~T} 25$, \\
\hline \multirow{2}{*}{$\begin{array}{l}\text { Contexto de } \\
\text { Aprendizagem }\end{array}$} & \multirow{2}{*}{12} & $\begin{array}{r}\text { Preferências de uso e interação dos objetos de } \\
\text { aprendizagem }\end{array}$ & 7 & $\mathrm{~T} 4, \mathrm{~T} 11, \mathrm{~T} 12, \mathrm{~T} 20, \mathrm{~T} 32^{\star} \mathrm{T}, \mathrm{T} 43$, \\
\hline & & $\begin{array}{l}\text { Atendimento às necessidades dos alunos e reduçãa de } \\
\text { evasãa }\end{array}$ & 5 & $\mathrm{~T} 13, \mathrm{~T} 18, \mathrm{~T} 35, \mathrm{~T} 44, \mathrm{~T} 45$ \\
\hline \multirow{3}{*}{$\begin{array}{l}\text { Customização de } \\
\text { conteúdo/ambientes } \\
\text { virtuais }\end{array}$} & \multirow{3}{*}{9} & $\begin{array}{l}\text { Design Educacional de objetos de aprendizagem } \\
\text { baseado nas preferências dos estudantes }\end{array}$ & 3 & $\mathrm{~T} 5, \mathrm{~T} 38, \mathrm{~T} 41$ \\
\hline & & $\begin{array}{r}\text { Analítica de aprendizagem (Learming Analytics) e } \\
\text { customização de ambientes virtuais }\end{array}$ & 3 & $\mathrm{~T} 7, \mathrm{~T} 22, \mathrm{~T} 28$ \\
\hline & & $\begin{array}{r}\text { Material Didático e Ambiente Virtual adaptativos } \\
\text { (Adaptive Learning) }\end{array}$ & 3 & $\mathrm{~T} 9, \mathrm{~T} 30, \mathrm{~T} 31, \mathrm{~T}_{2} 2^{*}$ \\
\hline
\end{tabular}

Após o fichamento das produções e as observações geradas na categorização por focos temáticos, foi possível perceber a aplicação de instrumentos para diagnosticar os estilos de aprendizagem dos estudantes em seus respectivos cursos, como um enfoque recorrente nos trabalhos mapeados na triangulação dos descritores apresentados na escolha metodológica (Figura III).

Conectado aos objetivos deste estudo, não pretende-se, nesse momento, detalhar os focos gerados, mas perceber elementos que possam evidenciar cenários de investigação da temática proposta neste estudo.

[...]A vantagem dessa forma de organização é que ela permite comparar por contraste os diferentes olhares e resultados produzidos, independendo da opção teórica ou metodológica de cada estudo. Isso não significa 
ecletismo. Significa, acima e tudo, respeito à diversidade e às múltiplas formas de produzir conhecimentos dentro de um campo específico. (Fiorentini, 2002, p.5)

Diante dos resultados, na Figura 3 há o registro da convergência dos descritores (Estilos de Aprendizagem, Ambiente Virtual, Educação Superior) acerca das percepções obtidas na categorização e distribuição das publicações nos focos e subfocos temáricos. De forma sintética, consideram-se: o reconhecimento do perfil para uma determinada área/curso, práticas de utilização de recursos e formas de interação percebidas nas preferências dos usuários e estratégias de ensino para motivar os estudantes, como aspectos característicos do cenário de investigações que recorreram à abordagem das teorias de estilo de aprendizagem em cursos superiores suportado por tecnologias digitais.

FigURA III - Mapeamento dos focos temáticos

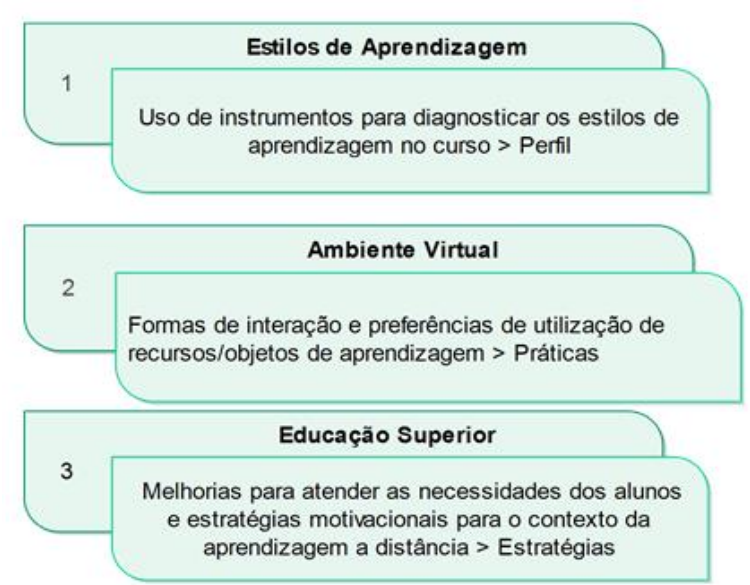

\section{CONSIDERAÇÕES FinAIS}

Os resultados obtidos neste estudo demonstraram que o mapeamento em torno das produções selecionadas trouxe à tona uma reflexão oportuna quanto ao uso de teorias do estilo de aprendizagem para o cenário da educação superior suportado por ambientes virtuais.

A categorização dos focos e subfocos temáticos, oportuniza caminhos para o aprofundamento de novos estudos para explorar estratégias que favorecem experiências inovadoras de aprendizagem. A análise e fichamento das leituras subsidiaram as conexões entre a triangulação dos dados e os focos temáticos percebidos durante o mapeamento. Tal fato, possibilita dizer que embora as inovações tecnológicas tenham impactado consideravelmente no campo da educação, todo e qualquer modelo pedagógico a ser adotado não deve repensar as suas práticas sem considerá-las, pois a relação entre ensinar e aprender convivem lado a lado e estão passíveis de inversão, à medida que novas formas de contato com o conhecimento são percebidas.

Recorrer aos estilos de aprendizagem demonstra uma preocupação em traçar perfis, melhorar práticas e oportunizar ações pedagógicas que estejam conectadas às preferências e a realidade discente.

Dentre as possibilidades futuras de investigações, pode-se destacar a necessidade de intensificar e direcionar novos estudos para o uso de ambientes virtuais adaptativos tendo em vista a carência encontrada nos trabalhos selecionados quando relacionados aos estilos de aprendizagem e personalização de conteúdos no contexto de cursos superiores a distância. 


\section{REFERÊNCIAS}

Alonso, C. M., Gallego, D. J., \& Honey, P. (2002). Los estilos de aprendizaje: procedimientos de diagnóstico y mejora. madrid: mensajero.

Barros, D. M. V. (2009). Estilos de uso do espaço virtual: Como se aprende e se ensina no virtual?. Revista Inter-ação, 51-74.

Barros, D. M. (2011). Estilos de aprendizagem na atualidade.

Belloni, M. L. (1999). Professor coletivo: Quem ensina a distância. Educação a Distância. Campinas: Autores Associados.

Brasil - Ministério da Educação Secretaria de Educação a Distância.. (2007). Referenciais de Qualidade para a Educação Superior a Distância. Disponível em <http://portal.mec.gov.br/seed/arquivos/ pdf/legislacao/refead1.pd>

Censo EAD.BR, C. (2015). Relatório Analítico da Aprendizagem a Distância no Brasil. Curitiba: Ibpex.

DeAquino, C. T. (2007). Como aprender: Andragogia e as Habilidades de Aprendizagem. São Paulo: Pearson.

Dias, R. A., \& Leite, L. S. (2010). Educação a distância - Da legislação ao pedagógico. Petrópolis: Vozes.

Dunn, R., \& Dunn, K. Y. (1989). Learning Style Inventory (LSI).

Felder R. M., \&. S. (1988). Leaning and Teaching Styles in Engineering Education. Journal of Engineering Education.

Ferreira, N. S. (2002). As pesquisas denominadas estado da arte. Educação \& Sociedade. Campinas.

Fiorentini, D. (1994). Rumos da Pesquisa Brasileira em Educação Matemática. Campinas: FE/UNICAMP.
Fiorentini, D. (2002). Mapeamento e balanço dos trabalhos do GT-19 (Educação Matemática) no período de 1998 a 2001. 25 REUNIÃO ANUAL-Associação Nacional de Pós-Graduação e Pesquisa em Educação-ANPEd. Caxambu, 29.

Freitas, J. M. (2013). Estilos de aprendizagem no virtual: as preferências do discente do ensino superior a distância.

Galvis, A. H. (1992). Ingeniería de software educativo. Bogotá.

Kolb, D. A. (1984). Experiential Learning Experience as The Source of Learning and Development. Englewood Cliff: Prentice-Hall.

Leite Filho, G. A., Batista, I. V. C., Paulo Júnior, J., \& Siqueira, R. L. (2008). Estilos de aprendizagem $\mathrm{x}$ desempenho acadêmico-uma aplicação do teste de Kolb em acadêmicos no curso de ciências contábeis. In Congresso USP de Controladoria e Contabilidade (Vol. 8).

Lopes, W. M. (2002). ILS- inventário de estilos de aprendizagem de FelderSaloman: investigação de sua validade em estudantes universitários de Belo Horizonte. Florianópolis: Universidade Federal de Santa Catarina.

Maia, C., \& Mattar., J. (2007). ABC da EaD : a educação a distância hoje. São Paulo: Prentice Hall.

Masetto, M. T. (2003). Competência Pedagógica do Professor Universitário. $4^{a}$. Reimpressão. São Paulo: Ed Sammus editorial.

Ota, M. A. (2011). Contribuições teórico-metodológicas para produção de materiais didáticos: um estudo das aplicações computacionais para a construção de recursos de aprendizagem em ambientes virtuais. Instituto de Educação, ULHT. 
Prado, M. E., \& Almeida, M. E. (2003). Redesenhando estratégias na própria ação: formação do professor a distância em ambiente digital. Avercamp.

Romanowski, J. P., \& Ens, R. T. (2006). As pesquisas denominadas do tipo "Estado da Arte” em educação. Revista Diálogo Educacional.

Sanchez, L. (2011). Estilos de aprendizagem e planejamento de indicadores de qualidade para a retenção do aluno e diminuição da evasão na Educação a Distância. Lisboa.

Santos, A. A., \& Bariani, I. C. (1999). Estilos Cognitivos e Estilo de Aprendizagem. Petrópolis.

Varela, A. V. (2011). Estilos de aprendizagem e o ensino superior: potencializando a atitude científica. Estilos de Aprendizagem na atualidade, 1-17.

Vark-Learn. (2006). A Brief Biography of Neil D. Fleming. 


\section{APÊNDICE}

Publicações consultadas nos repositórios brasileiros e portugueses

\begin{tabular}{|c|c|c|c|}
\hline ANO & Título & Autor (es) & ID \\
\hline \multirow[t]{2}{*}{ 운 } & Estilos de aprendizagem e educação a distância: algumas perguntas e respostas?! & $\begin{array}{l}\text { Daniela Melaré Vieira Barros, Ana Márcia Zuliani Bianchi, } \\
\text { Juliana Souza Nunes, Lia Cavellucci, Sandra Cristina A. } \\
\text { Teodósio Santos Valadas. }\end{array}$ & $\mathrm{T} 1$ \\
\hline & Estilos de uso do espaço virtual: novas perspectivas para os ambientes de aprendizagem online & Daniela Melaré Vieira Barros & T2 \\
\hline \multirow[b]{2}{*}{$\overline{\check{\nu}}$} & Estilos de aprendizagem na atualidade & Barros, Daniela Melaré Vieira & T3 \\
\hline & Explorando os estilos de aprendizagem em curso a distancia sobre o exame de papanicolaou & $\begin{array}{c}\text { José Maria Chagas Zanetti, Daniela Melaré Vieira Barros, Maria } \\
\text { Helena Baena de Moraes Lopes }\end{array}$ & T4 \\
\hline \multirow{11}{*}{$\tilde{\check{N}}$} & $\begin{array}{l}\text { Contribuições teórico-metodológicas para produção de materiais didáticos : um estudo das aplicações } \\
\text { computacionais para a construção de recursos de aprendizagem em ambientes virtuais }\end{array}$ & Ota, Marcos Andrei & T5 \\
\hline & $\begin{array}{c}\text { "Coreografias e Estratégias Didáticas Online e suas Relações com os Enfoques e Estilos de Aprendizagem } \\
\text { Docentes e Discentes" }\end{array}$ & $\begin{array}{l}\text { Silva, Cristine Lucia da Silva ; Padilha, Maria Auxiliadora } \\
\text { Soares (Orientador) }\end{array}$ & T6 \\
\hline & $\begin{array}{l}\text { Ambientes virtuais de aprendizagem análise das arquiteturas pedagógicas do curso de bacharelado em } \\
\text { administração pública do cesad/ufs. }\end{array}$ & Givaldo Almeida dos Santos & $\mathrm{T} 7$ \\
\hline & Estilos de Coaprendizagem para uma coletividade aberta de pesquisa & $\begin{array}{l}\text { Barros, Daniela; Miranda, Luísa; Goulão, Maria; Henriques, } \\
\text { Susana; Morais, Carlos }\end{array}$ & T8 \\
\hline & $\begin{array}{l}\text { Uma abordagem estocástica baseada em aprendizagem por reforço para modelagem automática e dinâmica de } \\
\text { estilos de aprendizagem de estudantes em sistemas adaptativos e inteligentes para educação a distância }\end{array}$ & Fabiano Azevedo Dorça & T9 \\
\hline & Learning styles identification module for sakai environment & Alves, Paulo; Miranda, Luisa; Morais, Carlos; Alves, Evandro & $\mathrm{T} 10$ \\
\hline & Diversidade na Universidade: estilos de pensamento na resolução de problemas & Gonçalves, Inês Faria de Sousa & T11 \\
\hline & Estilos e estratégias de aprendizagem de estudantes universitários & Lisliê Lopes Vidal Silva & $\mathrm{T} 12$ \\
\hline & Coletividade aberta de pesquisa: os estilos de coaprendizagem no cenário online & Barros, Daniela Melaré Vieira; Okada, Alexandra; Kenski, Vani & $\mathrm{T} 13$ \\
\hline & Estilos de aprendizagem: uso do virtual pelos estudantes do ensino superior & Luísa Miranda, Carlos Morais, Fátima Goulão, Daniela Melaré & T14 \\
\hline & $\begin{array}{l}\text { Estilos De Aprendizagem e Desempenho em Educação a Distância: um Estudo } \\
\text { Empírico com Alunos das Disciplinas de Contabilidade Geral e Gerencial }\end{array}$ & $\begin{array}{l}\text { Daniel Ramos Nogueira, Márcia Maria dos Santos Bortolocci } \\
\text { Espejo, Luciano Gomes dos Reis, Simone Bernardes Voese }\end{array}$ & $\mathrm{T} 15$ \\
\hline \multirow{4}{*}{$\stackrel{\infty}{\Sigma}$} & Uso de espaço colaborativo virtual no IUSC I e a teoria dos estilos de aprendizagem: um relato em construção & Ribeiro, Renato Antunes & T16 \\
\hline & $\begin{array}{c}\text { Se(r) um leitor em um ambiente virtual e aprendizagem: a utilização do insólito como estratégia de leitura e escrita } \\
\text { no ensino superior }\end{array}$ & Carmem Lúcia Quintana Pinto & $\mathrm{T} 17$ \\
\hline & Contributo exploratório para uma abordagem digital inclusiva: o caso das universidades de ensino a distância & Moleirinho, Marina ; Malheiro, Sofia; Morgado, Lina & T18 \\
\hline & Estilos de aprendizagem para uma coletividade aberta de pesquisa & $\begin{array}{l}\text { Barros, Daniela Melaré Vieira; Henriques, Susana; Goulăo, } \\
\text { Fátima; Miranda, Luisa; Morais, Carlos } \\
\end{array}$ & T19 \\
\hline
\end{tabular}




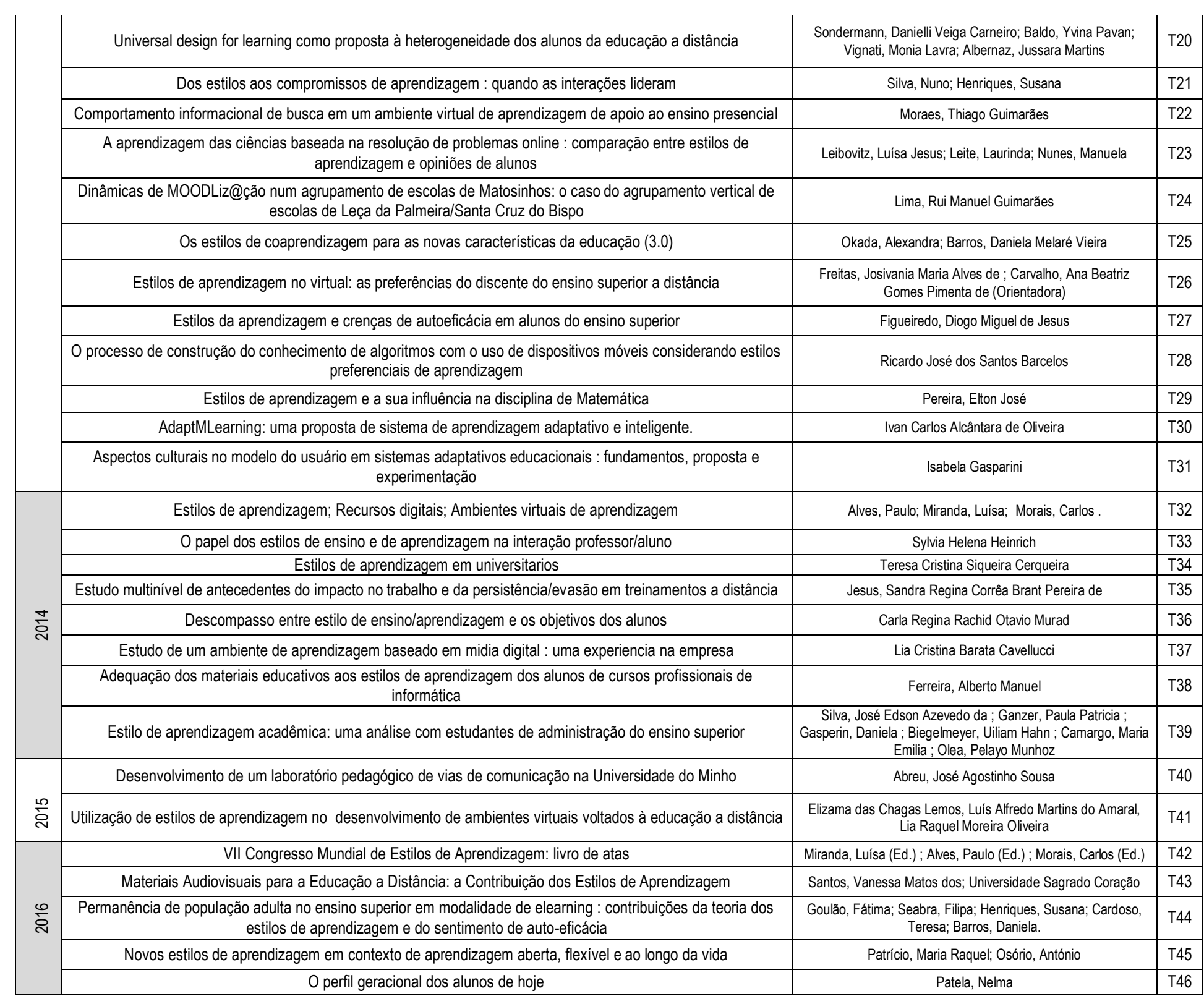


LEARNING STYLES IN VIRTUAL ENVIRONMENTS: RESEARCH SCENARIOS IN HIGHER EDUCATION

Abstract: This study proposes a brief investigation of academic and scientific papers in portuguese from 2010 to 2016 that have addressed the convergence of the fundamentals of learning style theories in virtual learning environments in the context of higher education.It is an exploratory study, with descritive analysis supported by bibliographic references.Data collection was carried out in the main portuguese and brazilian repositories. A total of 46 productions were found against the search criteria (title, subject and abstract) established. To compose the analysis of the results, the criterion of the organizational dimension and the categorization of the works by thematic focus and sub-focus was used. From the results, the mapping about investigations that dedicate to relate learning styles theories in the distance education scenario. Regarding the results, there is an effort in research that seeks to gather contributions and pedagogical practices that can approach the specific students needs enrolled in distance learning courses.

Keywords: Learning Styles; Virtual Environments; Distance Learning; Higher education; Research's mapping

\section{Texto:}

- Submetido: maio de 2017.

- Aprovado: junho de 2017.

\section{Para citar este artigo:}

Ota, M. A., Júnior, C. F. A., \& Barros, D. (2017). Estilos de aprendizagem em ambientes virtuais: cenários de investigação na educação superior. Educação, Formação \& Tecnologias, 10 (1), 47-58 [Online], disponível a partir de http://eft.educom.pt.

\section{Notas biográficas dos autores}

\section{i Marcos Andrei Ota}

Graduado em Pedagogia e Letras, especialização em Psicopedagogia, Tecnologias educacionais, Design Educacional e Educação a Distância. Mestrado em Ciências da Educação pela Universidade Lusófona de Humanidades e Tecnologias Lisboa, com pesquisa na área da educação e design educacional para produção de conteúdo didático em ambientes virtuais.

Atualmente é professor da Universidade Cidade de São Paulo e coordenador do núcleo de produção e desenvolvimento de materiais didáticos na Cruzeiro do Sul Virtual. Desenvolve pesquisas nas áreas de aprendizagem adaptativa (Doutorado), blended learning, learning design, mobile learning, Educação de Jovens e Adultos, estilos de aprendizagem em ambientes virtuais entre outros projetos com uso de tecnologias aplicadas ao ensino superior.

\section{ii Carlos Fernando Araujo Junior}

Brasileiro, professor pesquisador do Programa de Mestrado e Doutorado em Ensino de Ciências e Matemática, Universidade Cruzeiro do Sul. Atua na gestão acadêmica do Ensino Superior desde 1999. Atualmente é Pró-Reitor de Educação a Distância (Cruzeiro do Sul Educacional: Universidade Cruzeiro do Sul, Universidade Cidade de São Paulo -UNICID e Universidade de Franca- Unifran). Possui Graduação em Física pela Universidade Estadual Paulista Júlio de Mesquita Filho- UNESP/Rio Claro (1990), Mestrado em Física pelo Instituto de Física Teórica - IFT/UNESP (1993), Doutorado em Física Teórica pelo IFT/UNESP (1997) e MBA em Gestão Executiva pela Fundação Dom Cabral (FDC- Nova Lima/MG)

\section{iii Daniela Melaré Vieira Barros}

Brasileira / Italiana -Pedagoga USC, Especialista em Instrucional Designer, Especialista em Administração em Educação a Distância, Mestrado em Engenharia das Midias para a Educação Euromime- Erasmus Mundus- Portugal, Espanha e França, Mestrado em Educação pela UNESP- BRASIL, Doutorado em Educação UNESP - BRASIL, Pós - Doutorado pela UNICAMP e a UNED, Doutorado pela UNED de Madrid, Colaboradora da Open University no proyecto COLEARN. Professora Auxiliar Universidade Aberta 